Georgia State University

ScholarWorks @ Georgia State University

6-12-2015

\title{
Cumulative Neurological Factors Associated with Long-term Outcomes in Adult Survivors of Childhood Brain Tumors
}

Tricia Z. King

Georgia State University, tzking@gsu.edu

Sabrina $\mathrm{Na}$

Georgia State University, s.diana.na@gmail.com

Follow this and additional works at: https://scholarworks.gsu.edu/psych_facpub

Part of the Neuroscience and Neurobiology Commons, and the Psychology Commons

\section{Recommended Citation}

King, Tricia Z. and Na, Sabrina, "Cumulative Neurological Factors Associated with Long-term Outcomes in Adult Survivors of Childhood Brain Tumors" (2015). Psychology Faculty Publications. 151.

https://scholarworks.gsu.edu/psych_facpub/151

This Article is brought to you for free and open access by the Department of Psychology at ScholarWorks @ Georgia State University. It has been accepted for inclusion in Psychology Faculty Publications by an authorized administrator of ScholarWorks @ Georgia State University. For more information, please contact scholarworks@gsu.edu. 


\title{
Cumulative Neurological Factors Associated with Long-term Outcomes in Adult Survivors of Childhood Brain Tumors
}

\author{
Tricia Z. King*, Ph.D. \& Sabrina Na, B.S. \\ Department of Psychology \& Neuroscience Institute, Georgia State University, USA \\ ${ }^{*}$ Correspondence to: \\ Tricia Z. King, Ph.D., Department of Psychology and Neuroscience Institute, Georgia \\ State University, 140 Decatur Street, Urban Life Building 11th floor, Atlanta, GA 30303, \\ Tel: 404-413-6279; fax 404-413-6207; email: tzking@gsu.edu
}

Brief running title: Neurological Predictor Scale

Key words: brain tumors, long term survival, outcomes research, intelligence, adaptive functioning 


\begin{abstract}
Prior research has demonstrated the reliability and validity of the Neurological Predictor Scale (NPS) in relation to childhood brain tumor survivor outcomes; however, its use has not been examined in adult long-term survivors. The current study examines the concurrent validity of the NPS with long-term intellectual and adaptive outcomes in adult survivors of childhood brain tumors relative to individual variables alone. A total of 68 adult survivors of childhood brain tumors $(M=24$ years old, $S D=4)$ almost 16 years post diagnosis $(\mathrm{SD}=6)$ completed intellectual evaluations using the Wechsler Abbreviated Scale of Intelligence (WASI). Survivors' adaptive functioning skills were assessed via informant structured clinical interviews (SIB-R). NPS scores were computed from data acquired from medical records. The NPS was significantly associated with intellectual $\left(R^{2}=0.208, p<.05\right)$ and adaptive outcomes $\left(R^{2}=0.30, p<\right.$ .05) over and above individual risk factors. Approximately $18 \%$ of long-term survivors were identified as impaired in intellectual outcomes, and 29\% were identified as impaired in adaptive functioning in everyday life skills. The NPS quantifies the cumulative effects of treatment and neurological sequelae experienced by both short and long-term survivors of childhood brain tumors. It is a useful and easy measure to employ in clinical research that focuses on quantifying the neurological risk factors associated with long-term intellectual and adaptive functioning outcomes in adult survivors of childhood brain tumors.
\end{abstract}




\section{Cumulative Neurological Factors Associated with Long-term Outcomes in Adult Survivors of Childhood Brain Tumors}

\section{Introduction}

Modern multifaceted treatment regimens for childhood brain tumors have resulted in improved five-year survival rates (Ries, 2004) and a growing number of longterm survivors such that the majority of children diagnosed with brain tumors are now surviving into adulthood (Gurney et al., 2003). However, the risks for poor long-term outcomes are substantial, especially in those survivors treated aggressively and at a young age (Ellenberg et al., 2009; Mulhern, Merchant, Gajjar, Reddick, \& Kun, 2004). Studies have documented lower or suboptimal intellectual and adaptive outcomes in pediatric brain tumor survivors compared to their healthy peers (Beebe et al., 2005; Papazoglou, King, Morris, \& Krawiecki, 2008). Longitudinal studies have reported that these children are acquiring intellectual and adaptive skills at a slower rate relative to their same age peers but are not losing skills over time (Netson, Conklin, Wu, Xiong, \& Merchant, 2013; Palmer et al., 2001). By the time individuals are reaching adulthood, there are increased demands on and expectations of rapid development of cognitive abilities and independent living skills, but young adult survivors may experience more significant impairments relative to peers who are gaining these skills. Poor adult outcomes and neurocognitive impairment have primarily been documented via surveybased research by the Childhood Cancer Survivor Study (Ellenberg et al., 2009). Therefore, it is critical to better understand the neurological factors associated with long- 
term intellectual performance and adaptive outcomes in adult survivors of childhood brain tumors.

The research literature on long-term outcomes in adult survivors of brain tumors has identified numerous treatment factors and health risks that are associated with poor outcomes. Of these, radiation therapy is the most commonly reported predictor of poor outcomes, and this finding has been supported through a number of studies using various methodologies (e.g., radiation type, location, dose, age at radiation, time since radiation: (Armstrong et al., 2010; Armstrong et al., 2009; Mulhern, Hancock, Fairclough, \& Kun, 1992). Often a proportion of participants are administered radiation concurrently with chemotherapy as together these treatments contribute to increased survival, albeit with negative cognitive and adaptive outcomes. In addition, other factors such as hydrocephalus, seizures, and endocrine dysfunction have also been shown to contribute to late effects described in survivors (Anderson, 2003; Ater et al., 1996; Bruininks, Woodcock, Weatherman, \& Hill, 1996; Duffner, 2004, 2010; Hardy, Bonner, Willard, Watral, \& Gururangan, 2008; King et al., 2004; Ris \& Noll, 1994). These common neurological factors that influence outcomes are numerous, interactive and cumulative (Micklewright, King, Morris, \& Krawiecki, 2008; Roman \& Sperduto, 1995).

In a field with traditionally more limited sample sizes (Mulhern, Carpentieri, Shema, Stone, \& Fairclough, 1993; Ris \& Noll, 1994), examining these potential confounding and interrelated variables is complex and challenging. Ideally, clinical research would be able to employ multivariate modeling approaches to address the interactions between these important factors. However, the examination of multivariate interactions requires a sizeable sample in order to have enough power to detect 
significance, and smaller samples may present misleading results if interactions are not significant due to inadequate power and small effects. Similarly, in research designs when a large homogenous sample across each of these factors is not possible, examining the cumulative nature of the factors may help elucidate important contributions to outcomes. Although it is not as direct as examining multivariate interaction, it is important to consider not only the relation of each potential contributor to outcomes but also the cumulative neuro-oncology risk factors that are associated with the long-term outcomes of survivors of childhood brain tumors.

The Neurological Predictor Scale (NPS) provides researchers the ability to quantify the cumulative effects of tumor and treatment-related conditions (Micklewright et al., 2008). This measure takes into account the multiple treatments or neurological sequelae which the individual may have experienced. Studies have documented the reliability and concurrent validity in childhood survivors (Micklewright et al., 2008; Papazoglou, King, Morris, \& Krawiecki, 2009), but its concurrent validity has not yet been examined in adult survivors of childhood brain tumors. The current study aims to examine the concurrent validity of the NPS in adult survivors of childhood brain tumors.

\section{Methods}

\section{Participants and Procedures}

The study was reviewed and approved by the local institutional review boards, and all participants provided informed consent. Adult survivors were recruited through three different methods. First, adults who had been treated for a pediatric brain tumor at Children's Healthcare of Atlanta were identified. Second, survivors who had previously been part of a childhood brain tumor study were identified. Based on these two lists, a 
total of 676 letters were mailed; of these, 88 letters were returned due to an incorrect address. Finally, the Brain Tumor Foundation of Georgia ran an advertisement in their annual newsletter requesting participation in this study, which prompted other survivors to call to volunteer to participate in the study. Participants were excluded if English was not their first language, if they met criteria for pervasive developmental disorders, if they indicated a diagnosis of neurofibromatosis, or if they had experienced a traumatic brain injury (TBI). Notably, the sample included one participant who had experienced a stroke and five individuals who experienced posterior fossa syndrome following surgery. As these complications may contribute important neurological information associated with long-term outcomes, we did not exclude these individuals from the sample. However, to ensure that these individuals were not solely contributing to the results, the statistical analyses were rerun without these six individuals, and the results did not appreciably change. Therefore, the results presented include survivors who have experienced posterior fossa syndrome, as well as one person who has had a stroke. All participants were over the age of 18 and were at least five years past their most recent diagnosis in order to assess the effects of long-term survivorship.

Information about the brain tumor, subsequent treatments, and related neurological risk factors were obtained from a retrospective medical records review. We requested and reviewed physical medical records for all individuals who had been treated for their brain tumor before 2002 at Children's Healthcare of Atlanta. All medical encounters after 2002 were reviewed through an electronic health record software. For each participant in our study, we obtained information regarding tumor type (based on histology lab reports), tumor location (based on notes from the surgeon who had 
excised the tumor and MRI scan notes from radiologists) and all subsequent treatments (e.g. protocol numbers, type and dosage of radiation, chemotherapy, medications, type of surgery and dates surrounding these treatments). We also gathered information about comorbid neurological factors such as seizures, types of seizure medications, presence and types of hormone deficiency, presence of hydrocephalus and ensuing treatments for hydrocephalus. Data extraction agreement was $98 \%$ between raters. All discrepancies were discussed between the raters and resolved before being single- and double- entered into a database.

Overall, 68 participants were included in the analysis. The sample included 39 females and 29 males. Participants in the sample were between 18 and 35 years old, with an average age of 23.78 years $(S D=4.43)$. Additionally, participants were an average of 15.8 years post diagnosis $(S D=6.13)$. Tumor locations and tumor types were heterogeneous. About half of the sample was diagnosed with either medulloblastomas (29\%) or juvenile pilocytic astrocytomas (22\%); the remainder of the sample was diagnosed with one of 15 different types of brain tumors (See Table I). Brain tumor locations were also varied; the most common location for brain tumors was the posterior fossa (62\%), followed by the cerebral hemispheres (24\%). For a complete list of the characteristics of the sample, including but not limited to brain tumor type, location, and treatment variables, refer to Table I.

\section{Measures}

Wechsler Abbreviated Scale of Intelligence (WASI) 
The WASI is a nationally standardized abbreviated measure of intelligence comprised of four subtests. The measure has been shown to be reliable and valid, with high correlations with other established intelligence measures (Wechsler, 1999). Administration of all four subtests of this measure yields three indices: a verbal intelligence score (measuring expressive vocabulary, verbal knowledge and abstract verbal reasoning ability), a perceptual intelligence score (measuring spatial visualization, visual-motor coordination and nonverbal fluid reasoning), and an overall intellectual functioning score.

Scales of Independent Behavior - Revised (SIB-R)

The SIB-R is a norm-referenced assessment of adaptive and maladaptive behavior in school, home, employment, and community settings. Informants were interviewed by a trained graduate research assistant face-to-face. Informants were $82 \%$ parents, 9\% spouse/partner, 3\% grandparents/legal guardian, 3\% siblings and 3\% friends/roommates. Administration of this measure yields estimates of motor skills, social interaction/communication skills, personal living skills, community living skills, and broad independent living skills. These indices have demonstrated good reliability and validity in a number of populations (Bruininks et al., 1996).

\section{Neurological Predictor Scale (NPS)}

The NPS is a brief measure that incorporates tumor, tumor treatment, and other related neurological risk factors into one score. Prior to completing the NPS, two 
independent graduate students extracted information regarding treatments and commonly associated neurological risk factors from medical records.

These conditions and treatments were further coded into a corresponding number based on the NPS. As the items on the scale are objective ratings of treatments (i.e., presence or absence of treatments and conditions that are clearly and easily captured with a thorough medical records review) and subscores for each item on the scale are summed through a computer program, there was high agreement among raters $(100 \%)$. The measure has been validated with a small sample of child survivors of childhood brain tumors (Micklewright et al., 2008), and the purpose of the present study is to examine the concurrent validity of the scale in a larger sample of adult survivors of childhood brain tumors.

\section{Data Analysis}

First, we conducted a series of simple linear regression analyses. In these analyses, the NPS score was the independent variable, while intelligence measures (i.e., intellectual performance: verbal intelligence, perceptual intelligence) and adaptive functioning skill measures (i.e., broad independent living skills, motor skills, social communication skills, personal living skills and community living skills) were the dependent variables. This step was designed to ensure that the variance in NPS scores was significantly associated with the variance in the outcome measures in adult survivors of pediatric brain tumors.

Second, we conducted a series of hierarchical regression analyses to ascertain that the NPS score explains an additional amount of variance in two of the outcome 
measures (i.e., intellectual ability as indicated by the WASI and broad independent living skills) above and beyond the variance already explained by any individual factor. Individual treatment- or illness-related factors examined included type of radiation (i.e., no radiation, focal, whole brain, and whole brain with focal boost), presence of chemotherapy, presence of neurosurgery, presence of hydrocephalus, presence of hormone deficiency, and presence of seizure medications.

\section{Results}

A series of simple linear regression analyses indicated that the NPS scores were significantly associated with all of the dependent variables of interest. Specifically, the NPS scores were associated with intellectual performance, $\beta=-0.46, t(66)=-4.16, p<$ .001 , and $20.8 \%$ of the variance for intellectual ability $\left(\mathrm{R}^{2}=0.208, F(1,66)=17.33, p<\right.$ .01). The NPS scores also were also significantly associated with the participants' verbal intelligence scores, $\beta=-0.40, t(66)=-3.52, p<.001$, and perceptual intelligence scores, $\beta=-0.45, t(66)=-4.11, p<.001$. The NPS scores were associated with $15.8 \%$ of the variance for verbal intelligence $\left(\mathrm{R}^{2}=0.158, F(1,66)=12.42, p<.01\right)$ and $20.4 \%$ of the variance for perceptual intelligence scores $\left(\mathrm{R}^{2}=0.204, F(1,66)=16.92, p<.01\right)$.

The NPS scores were significantly associated with broad independent living skills, $\beta=-0.55, t(66)=-5.3, p<.001$, and accounted for $30 \%$ of the variance in broad independent living skills $\left(\mathrm{R}^{2}=0.30, F(1,66)=28.10, p<.001\right)$. The NPS scores were significantly associated with each of the component scores for adaptive functioning skills: motor ability $(\beta=-0.55, t(66)=-5.3, p<.001)$, social communication skills $(\beta=-$ 0.483, $t(66)=-4.48, p<.001)$, personal living skills $(\beta=-0.47, t(66)=-4.32, p<.001)$ 
and community living skills $(\beta=-0.49, t(66)=-4.60, p<.001)$. The NPS scores were also associated with between $22 \%$ and $36 \%$ of the variance of the SIB-R subscale scores (see Table II for specific values). In sum, the NPS was significantly associated with our dependent variables of interest: verbal intelligence, perceptual intelligence and adaptive functioning skills.

A series of hierarchical multiple regression analyses were conducted to evaluate whether the NPS scores accounted for a significant amount of variance above and beyond the contributions of individual treatment and illness-related variables (i.e., radiation, chemotherapy, neurosurgery, hydrocephalus, hormone deficiencies and seizure medications). The results indicated that the NPS scores accounted for a significant amount of the variance in overall intellectual functioning after controlling for the type of radiation $\left(R^{2} \Delta=0.076, p<0.05\right)$, presence of chemotherapy $\left(R^{2} \Delta=0.137, p\right.$ $<.05)$, presence of neurosurgery $\left(\mathrm{R}^{2} \Delta=0.222, p<.05\right)$, presence of hydrocephalus $\left(R^{2} \Delta=0.166, p<.05\right)$, presence of hormone deficiencies $\left(R^{2} \Delta=0.185, p<.05\right)$ and presence of seizure medications $\left(\mathrm{R}^{2} \Delta=0.208, p<.05\right)$. Similarly, the NPS scores accounted for a significant amount of the variance in broad independent living skills after controlling for individually examined treatment- and illness-related variables, including type of radiation $\left(\mathrm{R}^{2} \Delta=0.098, p<.05\right)$, presence of chemotherapy $\left(\mathrm{R}^{2} \Delta=\right.$ $0.161, p<.05)$, presence of neurosurgery $\left(\mathrm{R}^{2} \Delta=0.309, p<.05\right)$, presence of hydrocephalus $\left(\mathrm{R}^{2} \Delta=0.276, p<.05\right)$, presence of hormone deficiencies $\left(\mathrm{R}^{2} \Delta=0.232\right.$, $p<.05)$ and presence of seizure medications $\left(\mathrm{R}^{2} \Delta=0.298, p<.05\right)$. Table III displays additional details regarding each step of the hierarchical regressions and the change in adjusted $R^{2}$ associated with each step, as well as the standardized coefficients 
associated with each independent variable in the models. These analyses show that NPS scores were associated with intellectual and adaptive outcomes above and beyond each individual treatment or neurological risk factor.

In order to capture the diverse nature of the impact of treatments and tumor sequelae on outcomes, the inclusion of a heterogeneous tumor group was important for this study. Patients with rare diagnoses or other complicating conditions (stroke, posterior fossa syndrome) were examined in order to ensure that these participants were not appreciably influencing the association that we found between NPS scores and our dependent variables of interest. Examination of the scatterplots ensured that none of these rare cases were outliers. After removing the rare tumor cases and patients with complicating conditions (stroke, posterior fossa syndrome) from the analyses, the strength and directionality of the results did not appreciably change.

\section{Discussion}

The present study demonstrates that the NPS is more associated with intellectual and adaptive outcomes of childhood brain tumor survivors on average over 15 years post diagnosis than each independently examined individual treatment or neurological risk factor. The proportion of variance that the NPS accounted for ranged from 16 to $36 \%$ with medium-to-large effect sizes (0.4-0.6). These findings are consistent with past NPS studies on childhood brain tumor survivors (Micklewright et al., 2008; Papazoglou et al., 2009) suggesting that the NPS is a more robust measure of the complexity of neurological risk factors compared to one risk factor alone and that the NPS affords 
studies great power in detecting the role of cumulative neurological factors in long-term cognitive and adaptive outcomes.

The current findings also are consistent with previous literature on treatments (chemotherapy, radiation, neurosurgery) and neurological sequelae (hydrocephalus, seizure, and endocrine) (Anderson, 2003; Armstrong et al., 2009; Ater et al., 1996; Bruininks et al., 1996; Duffner, 2004, 2010; Hardy et al., 2008; King et al., 2004; Mulhern et al., 1992; Ris \& Noll, 1994; Roman \& Sperduto, 1995) being strongly associated with survivor outcomes. However, the current study demonstrates that the NPS is better than any one factor alone, as it takes into account the complexity and cumulative nature of neurological complications that are related to long-term outcomes in childhood brain tumor survivors.

Interestingly, survivors on average performed similar to peers on the WASI but a wide range of outcomes were evidenced. Indeed, $17.6 \%$ of the survivors were impaired on the intelligence measure $(z \leq-1.5)$. Similarly, adaptive outcomes were within normal limits on average but spanned an extremely wide range, and $29 \%$ of the sample was identified as impaired in the areas of adaptive functioning and day to day living skills on the SIB-R. The SIB-R is a structured clinical interview with informants who know the survivor well and observe him or her engaging in daily activities. This measure was even more robustly related to the NPS and identified a larger number of more significantly impaired survivors. This suggests that in spite of average intellectual performance, a sizeable proportion (29\%) of survivors is impaired in terms of everyday life skills. Furthermore, as neurological complications increase, it is more likely that impaired ability will be found in everyday function, which increases dependence on 
others. These findings are particularly important with young adult survivors whose peers are completing college or advanced education, securing employment or careers, developing and maintaining friendships and partners, and negotiating leisure activities in the community.

A greater proportion of impaired adaptive living skills may be related to another variable or variables, such as one or more impaired core cognitive skills. Disruption of core cognitive skills such as processing speed, attention, and/or working memory are commonly reported in the literature (Palmer, 2008; Reddick et al., 2003; Wolfe, MadanSwain, \& Kana, 2012) and may be better captured by the adaptive functioning skills in the SIB-R compared to the WASI. We specifically selected the WASI for the IQ measure so that these core cognitive skills would be less likely to depress estimates of global cognitive ability. Therefore the possibility of the NPS demonstrating concurrent validity with core cognitive skills should be examined in the future. In addition, these findings further support the benefit of obtaining complementary information about survivorship outcomes with both cognitive performance and informant report of everyday abilities.

It is important to note that treatment intensity has been examined to some extent in existing research literature. In these studies, the intensity variable is generally defined (in order from least to most intense) as stepwise groups of siblings, patients with acute lymphoblastic leukemia (ALL), patients with typical radiation with or without chemotherapy, and patients with a higher level of radiation with or without chemotherapy. However, modeling and measuring treatment intensity in this manner may not always be appropriate. Depending on the specific research question, how one quantifies treatment intensity or risk/prognostic factors is important. One could argue 
that a cumulative scale that takes into account factors common to brain tumor patients is superior to comparing across disease (e.g., ALL, brain tumor, and other cancer). For example, brain tumor patients share some extent of neurosurgery (subtotal, gross total, biopsy), hydrocephalus, or seizure medication. However, when comparing a radiation group to a no radiation group, participants may also differ on whether they received chemotherapy or if they had neuroendocrine involvement. Simply reporting that the findings are due to the neurotoxic effects of radiation may be misleading.

Acknowledging co-occurring factors and potential confounds as well as quantifying the cumulative nature of these differences as the NPS scores do may advance research in the field.

The NPS may be used as a tool for further examining research findings and parsing apart how treatment complexities relate to main research findings. For example, across radiation and no-radiation groups, one may examine how NPS scores or increased cumulative neurological risk are related to later reduced adaptive functioning (Papazoglou et al., 2009). The initial paper on the NPS (Micklewright et al., 2008) demonstrated concurrent validity in 25 short-term childhood brain tumor survivors with the NPS and IQ. Our research team also demonstrated that the NPS and parent report of attention problems was associated with later adaptive functioning in 42 children treated for brain tumors (Papazoglou et al., 2009). Another research team has recently demonstrated concurrent validity in an independent sample of 82 childhood brain tumor survivors on average 2.4 years since treatment, with the NPS and intellectual outcome (McCurdy, Rane, Daly, Jacobson, 2014). Similarly, another study examined an independent sample of 75 childhood brain tumor survivors on average 2.5 years since 
treatment, and found that poorer processing speed and working memory outcomes were associated with the NPS (McCurdy, 2014). In addition, our research team recently demonstrated that the NPS was specifically related to hippocampal and putamen volume but not associated with whole brain volume in 35 adult survivors of childhood brain tumors (Jayakar, King, Morris, \& Na, 2015). NPS findings such as these help to promote ideas for future larger scale research studies and expand the focus of research beyond radiation treatment alone. Although radiation treatment is a critical factor in outcome research, it may be misleading if complementary analyses of neurological complexity are not also considered. Therefore, the NPS appears to be associated with outcomes across different types of measurement without shared method variance, suggesting strong concurrent validity in both child and adult survivors of childhood brain tumors.

The current study should be considered in the context of its limitations. Participants were recruited through different mechanisms (e.g., letters and newsletters) making it difficult to know the true source population. Therefore, there may be selection bias in those participants who contacted the research team to participate in the study. It may be that the survivors who wanted to participate were more impaired and wanted more health care provider contact. It is equally possible, in this convenience sample, that the survivors who wanted to participate were more able to given higher cognitive or adaptive functioning skills. Another limitation that should be considered is that the survivors in this study were treated between 1979 and 2008 and therefore are unlikely to reflect the most recent treatment advances being implemented. That said, the growing number of survivors requires multidisciplinary teams in order to continue 
considering the role of these cumulative risk factors from older treatment practices in long-term outcomes. It also is recognized that some study designs may have a precise question about the role of radiation dosages to specific brain areas in specific diagnostic groups. In this situation, highly homogenous diagnostic and radiation treatment data is necessary. However, to rule out other potential confounds that may be concurrently contributing to the outcomes observed in that hypothetical homogenous group, researchers may consider using the NPS to quantify other complex medical factors that may differ between participants and identify how much additional variance these related factors may also contribute. While the NPS is not able to contribute to specific hypotheses that require a refined measurement strategy such as dosimetry or integral biologically effective dose (IBED:(Ris, 2007) when examining the impact of radiation on cognitive outcome, it may provide complementary information in the same study about the association between the cumulative risk and outcomes, indicating the other potentially important co-occurring complications. Therefore, the NPS has the potential to contribute to our knowledge of complex risk factors in survivors of childhood brain tumors. Future prospective longitudinal studies with larger samples may consider exploring if the cumulative NPS scale adds complementary information to multivariate analyses.

In any location in the world, the sample size of children undergoing treatment for brain tumor is fortunately not large. In order to capture the multiple potential complications a survivor may experience, a cumulative measure such as the NPS may be the easiest to implement. The NPS as it is currently designed is not able to examine the interactions that may exist between individual treatment factors, but it is certainly a 
first step toward examining neurological complexity within brain tumor samples above and beyond simple descriptive reports of each variable.

In summary, the current study demonstrates the concurrent validity between the NPS and intellectual and adaptive functioning measures. Based on these results, the NPS may be an informative clinical research tool especially in studies with heterogeneous brain tumor samples. Notably, the NPS is very user-friendly; the scale is clear, straightforward and efficient to complete after obtaining thorough medical record review information. Future research could examine the role of these factors when examining neurobiological variables (e.g., white matter volume and microstructure), core cognitive (e.g., processing speed, attention and working memory) and psychosocial outcomes, quality of life, and other factors critical to improving long-term cancer survivorship. 
Acknowledgements This research was supported by a Research Scholar Grant from the American Cancer Society (PI: T.Z.K., \#RSGPB-CPPB-114044) and Georgia State University Brains and Behavior Initiative Graduate Student Fellowship (S.N.). We would like to express our gratitude to the individuals and families who participated in this study and generously contributed their time and effort. Also, we would like to acknowledge the King Developmental Neuropsychology Research Team for helping with data acquisition, including but not limited to, Drs. Jackie Micklewright, Matthew Mumaw, Aimilia Papazoglou, \& John Ryan; and Kristen Smith, Ryan Brewster, Alyssa Aillion, and Michelle Fox.

Conflict of Interest: Tricia King and Sabrina Na declare that they have no conflict of interest. 


\section{References}

Anderson, N. E. (2003). Late complications in childhood central nervous system tumour survivors. Curr Opin Neurol, 16(6), 677-683. doi: 10.1097/01.wco.0000102623.38669.e5

Armstrong, G. T., Jain, N., Liu, W., Merchant, T. E., Stovall, M., Srivastava, D. K., . . Krull, K. R. (2010). Region-specific radiotherapy and neuropsychological outcomes in adult survivors of childhood CNS malignancies. Neuro Oncol, 12(11), 1173-1186. doi: 10.1093/neuonc/noq104

Armstrong, G. T., Liu, Q., Yasui, Y., Huang, S., Ness, K. K., Leisenring, W., . . Packer, R. J. (2009). Longterm outcomes among adult survivors of childhood central nervous system malignancies in the Childhood Cancer Survivor Study. J Nat/ Cancer Inst, 101(13), 946-958. doi: 10.1093/jnci/djp148

Ater, J. L., Moore, B. D., 3rd, Francis, D. J., Castillo, R., Slopis, J., \& Copeland, D. R. (1996). Correlation of medical and neurosurgical events with neuropsychological status in children at diagnosis of astrocytoma: utilization of a neurological severity score. J Child Neurol, 11(6), 462-469.

Beebe, D. W., Ris, M. D., Armstrong, F. D., Fontanesi, J., Mulhern, R., Holmes, E., \& Wisoff, J. H. (2005). Cognitive and adaptive outcome in low-grade pediatric cerebellar astrocytomas: evidence of diminished cognitive and adaptive functioning in National Collaborative Research Studies (CCG 9891/POG 9130). J Clin Oncol, 23(22), 5198-5204. doi: 10.1200/JCO.2005.06.117

Bruininks, R. H., Woodcock, R. W., Weatherman, R. F., \& Hill, B. K. (1996). Scales of Independent Behavior--Revised: Riverside Publishing.

Duffner, P. K. (2004). Long-term effects of radiation therapy on cognitive and endocrine function in children with leukemia and brain tumors. Neurologist, 10(6), 293-310. doi: 10.1097/01.nrl.0000144287.35993.96

Duffner, P. K. (2010). Risk factors for cognitive decline in children treated for brain tumors. Eur J Paediatr Neurol, 14(2), 106-115. doi: 10.1016/j.ejpn.2009.10.005

Ellenberg, L., Liu, Q., Gioia, G., Yasui, Y., Packer, R. J., Mertens, A., ... Zeltzer, L. K. (2009). Neurocognitive status in long-term survivors of childhood CNS malignancies: a report from the Childhood Cancer Survivor Study. Neuropsychology, 23(6), 705-717. doi: 10.1037/a0016674

Gurney, J. G., Kadan-Lottick, N. S., Packer, R. J., Neglia, J. P., Sklar, C. A., Punyko, J. A., . . Robison, L. L. (2003). Endocrine and cardiovascular late effects among adult survivors of childhood brain tumors: Childhood Cancer Survivor Study. Cancer, 97(3), 663-673. doi: 10.1002/cncr.11095

Hardy, K. K., Bonner, M. J., Willard, V. W., Watral, M. A., \& Gururangan, S. (2008). Hydrocephalus as a possible additional contributor to cognitive outcome in survivors of pediatric medulloblastoma. Psychooncology, 17(11), 1157-1161. doi: 10.1002/pon.1349

Jayakar, R., King, T. Z., Morris, R., \& Na, S. (2015). Hippocampal volume and auditory attention on a verbal memory task with adult survivors of pediatric brain tumor. Neuropsychology, 29(2), 303319. doi: $10.1037 /$ neu0000183

King, T. Z., Fennell, E. B., Williams, L., Algina, J., Boggs, S., Crosson, B., \& Leonard, C. (2004). Verbal memory abilities of children with brain tumors. Child Neuropsychol, 10(2), 76-88. doi: 10.1080/09297040490911096

McCurdy, M. D., Padden, E., Rane, S., Daly, B.P., \& Jacobson, L.A. . (2014). Predicting neurocognitive and neurobehavioral outcome following childhood brain tumor. The Clinical Neuropsychologist, 27(5).

Micklewright, J. L., King, T. Z., Morris, R. D., \& Krawiecki, N. (2008). Quantifying pediatric neuro-oncology risk factors: development of the neurological predictor scale. J Child Neurol, 23(4), 455-458. doi: 10.1177/0883073807309241

Mulhern, R. K., Carpentieri, S., Shema, S., Stone, P., \& Fairclough, D. (1993). Factors associated with social and behavioral problems among children recently diagnosed with brain tumor. $J$ Pediatr Psychol, 18(3), 339-350. 
Mulhern, R. K., Hancock, J., Fairclough, D., \& Kun, L. (1992). Neuropsychological status of children treated for brain tumors: a critical review and integrative analysis. Med Pediatr Oncol, 20(3), 181-191.

Mulhern, R. K., Merchant, T. E., Gajjar, A., Reddick, W. E., \& Kun, L. E. (2004). Late neurocognitive sequelae in survivors of brain tumours in childhood. Lancet Oncol, 5(7), 399-408. doi: 10.1016/S1470-2045(04)01507-4

Netson, K. L., Conklin, H. M., Wu, S., Xiong, X., \& Merchant, T. E. (2013). Longitudinal investigation of adaptive functioning following conformal irradiation for pediatric craniopharyngioma and lowgrade glioma. Int J Radiat Oncol Biol Phys, 85(5), 1301-1306. doi: 10.1016/j.ijrobp.2012.10.031

Palmer, S. L. (2008). Neurodevelopmental impact on children treated for medulloblastoma: a review and proposed conceptual model. Dev Disabil Res Rev, 14(3), 203-210. doi: 10.1002/ddrr.32

Palmer, S. L., Goloubeva, O., Reddick, W. E., Glass, J. O., Gajjar, A., Kun, L., . . Mulhern, R. K. (2001). Patterns of intellectual development among survivors of pediatric medulloblastoma: a longitudinal analysis. J Clin Oncol, 19(8), 2302-2308.

Papazoglou, A., King, T. Z., Morris, R. D., \& Krawiecki, N. (2009). Parent report of attention problems predicts later adaptive functioning in children with brain tumors. Child Neuropsychol, 15(1), 4052. doi: 10.1080/09297040802036102

Papazoglou, A., King, T. Z., Morris, R. D., \& Krawiecki, N. S. (2008). Cognitive predictors of adaptive functioning vary according to pediatric brain tumor location. Dev Neuropsychol, 33(4), 505-520. doi: $10.1080 / 87565640802101490$

Reddick, W. E., White, H. A., Glass, J. O., Wheeler, G. C., Thompson, S. J., Gajjar, A., . . Mulhern, R. K. (2003). Developmental model relating white matter volume to neurocognitive deficits in pediatric brain tumor survivors. Cancer, 97(10), 2512-2519. doi: 10.1002/cncr.11355

Ries, L., Eisner ,MP, Kosary, CL, Hankey, BF, Miller, BA, Clegg, L, Mariotto, A, Feuer, EJ, Edwards, BK (Eds). (2004). SEER Cancer Statistics Review, 1975-2001. Bethesda, MD

National Cancer Institute

Ris, M. D. (2007). Lessons in pediatric neuropsycho-oncology: what we have learned since Johnny Gunther. J Pediatr Psychol, 32(9), 1029-1037. doi: 10.1093/jpepsy/jsm018

Ris, M. D., \& Noll, R. B. (1994). Long-term neurobehavioral outcome in pediatric brain-tumor patients: review and methodological critique. J Clin Exp Neuropsychol, 16(1), 21-42. doi: 10.1080/01688639408402615

Roman, D. D., \& Sperduto, P. W. (1995). Neuropsychological effects of cranial radiation: current knowledge and future directions. Int J Radiat Oncol Biol Phys, 31(4), 983-998. doi: 10.1016/0360-3016(94)00550-8

Wechsler, D. (1999). Wechsler Abbreviated Scale of Intelligence (WASI) Manual. San Antonio, TX: Psychological Corporation.

Wolfe, K. R., Madan-Swain, A., \& Kana, R. K. (2012). Executive dysfunction in pediatric posterior fossa tumor survivors: a systematic literature review of neurocognitive deficits and interventions. Dev Neuropsychol, 37(2), 153-175. doi: 10.1080/87565641.2011.632462 
Table I. Demographic, Diagnostic and Treatment Variables.

\begin{tabular}{|c|c|}
\hline Variables & Survivors $(n=68)$ \\
\hline \multicolumn{2}{|l|}{ Demographic Variables } \\
\hline Female (n, \%) & $39(57 \%)$ \\
\hline Hollingshead socio-economic status (mean \pm SD) & $2.2 \pm 1.09$ \\
\hline Age at testing (mean $\pm S D)$ & $23.78 \pm 4.43$ \\
\hline Range & $18-35$ \\
\hline \multicolumn{2}{|l|}{ Ethnicity (n, \%) } \\
\hline Caucasian & $55(81 \%)$ \\
\hline African-American & $9(13 \%)$ \\
\hline Other & $4(6 \%)$ \\
\hline \multicolumn{2}{|l|}{ Employment status (n, \%) } \\
\hline Student & $31(46 \%)$ \\
\hline Employed & $22(32 \%)$ \\
\hline Unemployed & $8(12 \%)$ \\
\hline Disability & $7(10 \%)$ \\
\hline \multicolumn{2}{|l|}{ Diagnostic Variables } \\
\hline Years post diagnosis (mean $\pm S D$ ) & $15.8 \pm 6.13$ \\
\hline Range & $5-28$ \\
\hline Age at diagnosis (mean $\pm S D$ ) & $7.96 \pm 4.8$ \\
\hline Range & $0-17$ \\
\hline \multicolumn{2}{|l|}{ Tumor location $(n, \%)$} \\
\hline Posterior fossa & $42(62 \%)$ \\
\hline Cerebral hemispheres & $16(24 \%)$ \\
\hline Ventricles & $5(7 \%)$ \\
\hline Pituitary & $3(4 \%)$ \\
\hline Brain stem & $2(3 \%)$ \\
\hline \multicolumn{2}{|l|}{ Tumor type $(n, \%)$} \\
\hline Medulloblastoma & $20(29 \%)$ \\
\hline Juvenile pilocytic astrocytoma & $15(22 \%)$ \\
\hline Diffuse astrocytoma & $6(9 \%)$ \\
\hline Craniopharyngioma & $5(7 \%)$ \\
\hline Ganglioglioma & $3(4 \%)$ \\
\hline Ependymoma & $3(4 \%)$ \\
\hline Other $^{\dagger}$ & $16(25 \%)$ \\
\hline \multicolumn{2}{|l|}{ Treatment Variables } \\
\hline Radiation ( $n, \%)$ & $35(52 \%)$ \\
\hline \multicolumn{2}{|l|}{ Radiation type (n, \%) } \\
\hline Focal & $8(23 \%)$ \\
\hline Whole brain & $4(11 \%)$ \\
\hline
\end{tabular}


Craniospinal with boost

Chemotherapy $(n, \%)$

Neurosurgery

Hydrocephalus ( $n, \%)$

Seizure Medication $(n, \%)$

Hormone deficiency $(n, \%)$

Hormone deficiency type (n, \%)

Hypothyroidism

Growth hormone deficiency

Hypogonadism

Hypoadrenalism

\section{Measures}

Full scale IQ (mean $\pm S D)$

Range

$\%$ impaired (s-1.5z)

Broad independent living skills standard score

(mean \pm SD)

Range

$\%$ impaired ( $\leq-1.5 z)$
$23(66 \%)$

$25(37 \%)$

67 (99\%)

$46(68 \%)$

$18(27 \%)$

$33(49 \%)$

$22(32 \%)$

$15(22 \%)$

$6(9 \%)$

$5(7 \%)$

$95.53 \pm 18.79$

$54-128$

$17.6 \%$

$95.24 \pm 28.98$

$33-144$

$29.4 \%$

Note. ${ }^{\dagger}$ Two oligodendroglioma, two brainstem glioma, two anaplastic astrocytoma, two cerebral neuroblastoma, two mixed astrocytoma/ganglioglioma, one malignant melanoma, one pineoblastoma, one meningioma, one germ cell tumor, one medulloepithelioma, one non-infiltrative mesenchymal neoplasm, not otherwise specified.

Table II. Simple Linear Regression Models of the Predictive Relationship Between NPS and Various Dependent Variables of Interest $(n=68)$

\begin{tabular}{|c|c|c|c|c|c|}
\hline Measure & $B$ & SE B & $\beta$ & $t$ & $p$ \\
\hline Full Scale IQ & -3.81 & 0.92 & -.46 & -4.16 & $<.001^{\star *}$ \\
\hline Verbal IQ & -3.08 & 0.87 & -.40 & -3.52 & $.001^{* *}$ \\
\hline Perceptual IQ & -3.76 & 0.91 & -.45 & -4.11 & $<.001^{\star *}$ \\
\hline Broad Independent Living & -7.04 & 1.33 & -.55 & -5.30 & $<.001^{* *}$ \\
\hline \multicolumn{6}{|l|}{ Skills } \\
\hline Motor Skills & -8.85 & 1.44 & -.60 & -6.15 & $<.001^{* *}$ \\
\hline Social Communication Skills & -4.93 & 1.10 & -.48 & -4.48 & $<.001^{* *}$ \\
\hline Personal Living Skills & -4.69 & 1.09 & -.47 & -4.32 & $<.001^{\star *}$ \\
\hline Community Living Skills & -5.42 & 1.18 & -.49 & -4.60 & $<.001^{* *}$ \\
\hline
\end{tabular}

Note. ${ }^{\star} p<.05,{ }^{* *} p<.01$ 
Table III Hierarchical Regression Models Relating Intelligence and Broad Independent Living Skills to Treatment Related Factors and NPS Scores ( $\mathrm{N}=68)$

$$
\text { Intellectual Functioning Broad Independent Living }
$$
Skills

Individual Treatment Factor

$\underset{R^{2}}{\text { Adjusted }} \quad \Delta \mathrm{R}^{2} \quad \beta \quad \underset{R^{2}}{\text { Adjusted }} \quad \Delta \mathrm{R}^{2}$

$\beta$

\section{Radiation}

Model 1

Type of Radiation

Model 2

.12

.13

.18

.19

Type of Radiation

.18

.08

$-.36^{* *}$

.27

$-.44^{* *}$

NPS Score

.07

.10

$-.51^{\star}$

.05

Chemotherapy

Model 1

.07

Presence of Chemotherapy

Model 2

Presence of Chemotherapy

NPS Score

.08

$-.29^{*}$

.20

.14

.18

.13

.14

$-.58^{* *}$

Neurosurgery

Model 1

Presence of Neurosurgery

Model 2

Presence of Neurosurgery

NPS Score

Hydrocephalus

Model 1

Presence of Hydrocephalus

Model 2

Presence of Hydrocephalus

NPS Score

$-.02$

.0

$-.015$

.0

.017

.20

.22

.29

$-.02$

.12

$-.48^{\star *}$

.16

$-.38^{* *}$

$.593^{* *}$

$-.64^{\star *}$

\section{Hormone Deficiencies}

Model 1

Presence of Hormone Deficiencies

Model 2

Presence of Hormone Deficiencies

NPS Score

.04

.05

$-.23$

.01

.02

.20

.17

.28

$-.15$

$-.11$

$-.42^{\star *}$

.28

.11

$-.57^{\star \star}$

\section{Seizure Medication}

Model 1

Presence of Seizure Medications

Model 2

Presence of Seizure Medications

NPS Score

$\begin{array}{llll}-.003 & .012 & -.114 & .10\end{array}$

.28

$-.34^{\star *}$

$.22 \quad .23$

.23

$-.59^{\star *}$

$-.04$

$-.53^{* *}$

.0

$-.02$

$-.02$

.0

.18

.21

$-.017$

$-.02$

$-.006$

.28

.30

.002

$-.55^{\star *}$

Note. ${ }^{*} p<.05,{ }^{* *} p<.01$ All beta values for NPS scores in the second models for both dependent variables were significant. This finding indicates that NPS continues to predict intellectual and adaptive outcomes after controlling for individual risk factors. 